\title{
A NEW METHOD IN FIXED POINT THEORY
}

\author{
BY RICHARD G. SWAN ${ }^{1}$
}

Communicated by S. Eilenberg, February 10, 1959

By modifying the construction of the Cartan-Leray spectral sequence of a covering [ 1 , Chapter $16, \S 8]$, it is possible to prove the following result.

Theorem 1. Let $\pi$ be a finite group acting on a space $X$. Let $A$ be a closed $\pi$-stable subspace of $X$. Then there is a convergent spectral sequence with

$$
E_{2}^{i, j}(X, A)=\hat{H}^{i}\left(\pi, H^{j}(X, A)\right)
$$

where $\hat{H}^{i}$ denotes the Tate cohomology of $\pi\left[1\right.$, Chapter 12] and $H^{i}(X, A)$ is the jth Cech cohomology group of $(X, A)$ with arbitrary coefficients and with $\pi$ action induced by that on $X$. The term $E_{\infty}$ is the graded module associated with a certain filtered module $J^{*}(X, A)$. This sequence is natural with respect to $\pi$-equivariant maps of $(X, A)$. If $\pi$ acts trivially on $X$, the sequence is trivial i.e. $E_{2}=E_{\infty}$.

In order to compute $J^{*}(X, A)$, we must impose rather drastic conditions.

THEOREM 2. Let $X$ be a paracompact Hausdorff space with the property that any (open) covering of $X$ has a finite dimensional refinement. Assume that any point of $X$ is either left fixed by all elements of $\pi$ or else is such that all its images under elements of $\pi$ are distinct.

Let $X^{\pi}, A^{\pi}$ be the sets of fixed points in $X$ and $A$ respectively. Then the inclusion $i:\left(X^{\pi}, A^{\pi}\right) \rightarrow(X, A)$ induces an isomorphism

$$
i^{*}: J^{*}(X, A) \rightarrow J^{*}\left(X^{\pi}, A^{\pi}\right) .
$$

REMARK. $i^{*}$ need not be an isomorphism of filtered modules i.e. $F^{k} J^{*}(X, A) \rightarrow F^{k} J^{*}\left(X^{\pi}, A^{\pi}\right)$ need not be onto for all $k$.

Now, assume that $\pi$ is cyclic of prime order $p$. The condition of Theorem 2 about the action of $\pi$ is then trivially satisfied. The spectral sequence is annihilated by multiplication by $p$, the order of $\pi$. Consequently, all terms are $Z_{p}$-modules.

Theorem 3. Let $(X, A)$ and $\pi$ satisfy the conditions of Theorem 2 with $\pi$ cyclic of prime order $p$. Then for all integers $k$ and $n$, and any coefficient group,

${ }^{1}$ This work was done while the author was a National Science Foundation fellow. 


$$
\sum \operatorname{dim} E_{2}^{i, j}\left(X^{\pi}, A^{\pi}\right) \leqq \sum \operatorname{dim} E_{2}^{i, j}(X, A)
$$

the sums being over those $(i, j)$ such that $i+j=n, j \geqq k$. Here "dim" means dimension as $Z_{p}$-module.

This follows trivially from Theorems 1 and 2.

If we take $Z_{p}$ as coefficient group and evaluate $E_{2}^{t, j}\left(X^{\pi}, A^{\pi}\right)$ by the universal coefficient theorem, we get generalizations of the inequalities of Floyd [2]. The Smith homology sphere theorem [4] follows immediately from this and an easy argument using the spectral sequence.

By taking $Z$ as coefficient group and using the universal coefficient theorem, we get the following theorem which generalizes some inequalities of Heller [3].

Theorem 4. Let $(X, A)$ and $\pi$ be as in Theorem 3. Assume that $\pi$ acts trivially on $H^{q}(X, A ; Z)$ for all $q$. Then, for all $k$,

$$
\sum \operatorname{dim} H^{q}\left(X^{\pi}, A^{\pi} ; Z_{p}\right) \leqq \sum \operatorname{dim} H^{q}\left(X, A ; Z_{p}\right)
$$

the sums being over all $q \geqq k$ with $q-k$ even.

It is also easy to prove Heller's equality $\chi_{\pi}(X, A)=\chi\left(X^{\pi}, A^{\pi}\right)[3]$ by using the periodicity of the Tate cohomology of a cyclic group $\pi$.

It is also possible to prove theorems about the local properties of $\left(X^{\pi}, A^{\pi}\right)$. More interesting, however, is the fact that cup products are easily introduced into the spectral sequence. This makes it possible to relate the cohomology rings of $(X, A)$ and $\left(X^{\pi}, A^{\pi}\right)$. For example, we can ask whether $H^{*}\left(X^{\pi}, A^{\pi}\right)$ will have the cohomology ring of a product of spheres whenever $H^{*}(X, A)$ does. (Note that in the theorems stated by Heller [3] concerning products of spheres, the word "product" should be replaced everywhere by the word "wedge." It is easy to give counterexamples to the theorems as stated.) The following theorem gives a partial answer to this question. It is remarkable that even this theorem becomes false if $X^{\pi}$ is not assumed to be connected.

Theorem 5. Let $X$ and $\pi$ satisfy the conditions of Theorem 2 with $A=\varnothing$ and $\pi$ cyclic of prime order $p$. Assume that $\pi$ acts trivially on $H^{*}(X ; Z)$ and $H^{*}\left(X ; Z_{p}\right)$. Assume also that $H^{*}\left(X ; Z_{p}\right)$ is isomorphic to $H^{*}\left(S^{2 m} \times S^{2 n} ; Z_{p}\right)$ as a ring. Here $2 m$ and $2 n$ are any even positive integers.

If $X^{\pi}$ is connected, then $H^{*}\left(X^{\pi} ; Z_{p}\right)$ is isomorphic as a group to 
$H^{*}\left(S^{2 r} \times S^{2 s} ; Z_{p}\right)$ where $2 r$ and $2 s$ are even and positive and $r \leqq m, s \leqq n$.

Furthermore, if $r \neq s$, then $H^{*}\left(X^{\pi} ; Z_{p}\right)$ is isomorphic to

$$
H^{*}\left(S^{2 r} \times S^{2 s} ; Z_{p}\right)
$$

as a ring.

\section{REFERENCES}

1. H. Cartan and S. Eilenberg, Homological algebra, Princeton, 1956.

2. E. E. Floyd, On periodic maps and the Euler characteristic of associated spaces, Trans. Amer. Math. Soc. vol. 72 (1952) pp. 138-147.

3. A. Heller, Homological resolutions of complexes with operators, Ann. of Math. vol. 60 (1954) pp. 283-303.

4. P. A. Smith, Fixed points of periodic transformations, Appendix B of Lefschetz, Algebraic topology, Amer. Math. Soc. Colloquium Publications, vol. 27, 1942.

University of Chicago

\section{A COMPLETE CHARACTERIZATION FOR EXTREME FUNCTIONALS}

BY R. C. BUCK ${ }^{1}$

Communicated by A. M. Gleason, February 12, 1959

1. Introduction. Let $E$ be a real linear space with a seminorm \|\| . Let $S$ be the set of linear functionals of norm less than or equal to 1 ; thus, $L \in S$ whenever $|L(x)| \leqq\|x\|$ for all $x \in E$. In many problems, it is important to know something about the extreme points of $S$; $L$ is extreme if we cannot write $L=\left(L^{\prime}+L^{\prime \prime}\right) / 2$ with $L^{\prime}$ and $L^{\prime \prime}$ distinct members of $S$. In this note, a new procedure will be developed for the study of a particular functional $L$; in particular, this will provide a surprisingly simple and useful characterization for the extreme functionals. With each $L$, we shall associate a closed subspace $V_{L}$ of $E$ in such a fashion that the relative "flatness" of $S$ at $L$ is determined by the size of $V_{L}$. In particular, $L$ is an extreme point of $S$ if and only if $V_{L}$ is all of $E$. The results are formulated for a seminormed space $E$ to allow their application to certain special cases of considerable interest. One such question is discussed in the last section where we look for extreme functionals in the class of those that vanish on a fixed subspace $M$.

2. Construction of $V_{L}$. An equivalent way to say that $L$ is extreme in $S$ is to say that a functional $\theta$ obeys $\|L \pm \theta\| \leqq 1$ only if $\theta=0$. Because we are dealing with a real space, this condition on $\theta$ can be rewritten as

${ }^{1}$ John Simon Guggenheim Fellow. 